EPJ Web of Conferences 75, 06009 (2014)

DOI: $10.1051 /$ epjconf/ 20147506009

(C) Owned by the authors, published by EDP Sciences, 2014

\title{
Effect of layer thickness ratio on magnetization reversal process in stacked media with high coercivity
}

\author{
A. Oyama ${ }^{1,}$, and R. Sugita ${ }^{1}$ \\ ${ }^{1}$ Ibaraki Univ., 4-12-1 Nakanarusawa-cho, Hitachi, Ibaraki 316-8511, Japan
}

\begin{abstract}
Effect of thickness ratio and interlayer exchange coupling on time-evolutional magnetization reversal process in stacked media with high coercivity was investigated by utilizing micromagnetic simulation. Regardless of the layer thickness ratio, for each stacked medium the magnetization reversal process is divided into three regions, namely the spin-flop rotation, the incoherent rotation and the coherent rotation along with increase of the interlayer exchange coupling constant $A_{\text {interlayer }}$. In order to get the incoherent rotation region which is suitable for the recording media, it is required that the $A_{\text {interlayer }}$ is between about $1.3 \times 10^{-7}$ and $2.2 \times 10^{-7}$ $\mathrm{erg} / \mathrm{cm}$ for the media with the layer thickness ratio of $1: 3$ and $3: 1$, and that one is between about $1.8 \times 10^{-7}$ and $2.5 \times 10^{-7} \mathrm{erg} / \mathrm{cm}$ for the media with the ratio near $1: 1$.
\end{abstract}

\section{Introduction}

The stacked media are still a strong candidate for achieving ultra-high recording density in hard disks with high coercivity [1], [2]. It is important to elucidate magnetization change in soft and hard layers of the stacked media at the time of recording, where interlayer exchange coupling between the layers has an essential role for the magnetization change [3] - [6]. The thickness of the soft layer is generally from about $1 / 5$ to $1 / 3$ of the hard layer in stacked media of commercial hard disks. On the other hand, one of proposed next-generation stacked media has thicker soft layer than the hard layer [7]. However, magnetization reversal process of such stacked medium has not been discussed sufficiently yet. In this study, effect of layer thickness ratio of the soft layer to the hard layer and the interlayer exchange coupling on the time-evolutional magnetization reversal process in the stacked media with high coercivity was investigated by utilizing micromagnetic simulation.

\section{Calculation method}

In order to investigate the magnetization reversal process in the stacked media, magnetic printing [8] was used as recording technique in the simulation. Fig. 1 shows the simulation model of the magnetic printing used in the study. In magnetic printing, first, the recording layer is magnetized in downward direction by applying the initial magnetic field along the perpendicular direction of recording layer. Then the master medium with a patterned magnetic layer corresponding to signal to record is in contact with the recoding layer, and after that, the

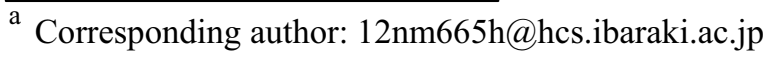

printing field is applied along the opposite direction to the initial magnetic field. Finally, the master pattern is printed onto the recording layer. The master pattern has the track width of $30 \mathrm{~nm}$ and the bit length of $30 \mathrm{~nm}$. Fig. 2 shows schematic of stacked media. Recording layer consists of the soft and hard layers. The

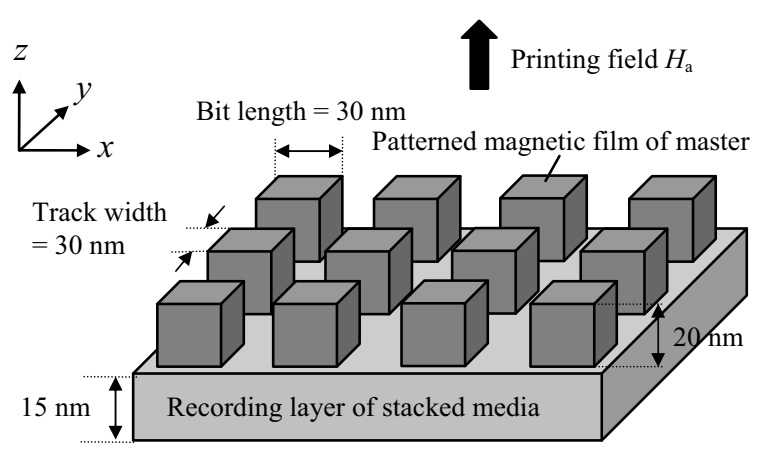

Fig. 1. Simulation model of magnetic printing.

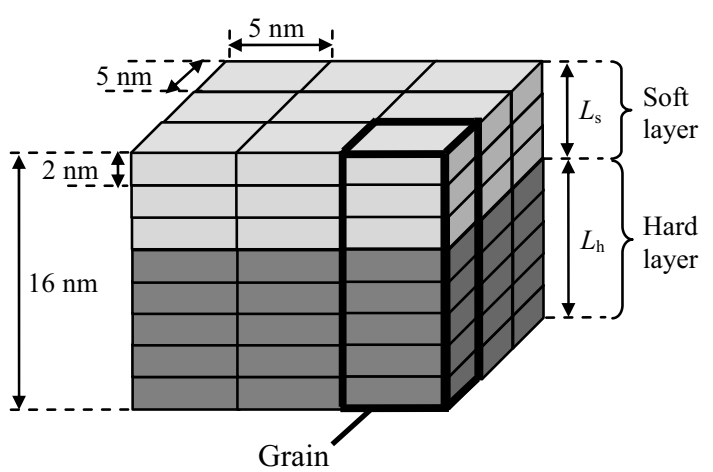

Fig. 2. Schematic of recording layer of stacked media. 
Table 1. Parameters of stacked media used in the study.

\begin{tabular}{|c|c|c|c|}
\hline \multicolumn{2}{|c|}{ Parameters } & Soft layer & Hard layer \\
\hline \multicolumn{2}{|c|}{ Thickness (nm) } & $L_{\mathrm{s}}$ & $L_{\mathrm{h}}\left(=16-L_{\mathrm{s}}\right)$ \\
\hline \multicolumn{2}{|c|}{$\begin{array}{l}\text { Saturation magnetization } \\
\qquad M_{\mathrm{s}}\left(\mathrm{emu} / \mathrm{cm}^{3}\right)\end{array}$} & \multicolumn{2}{|c|}{600} \\
\hline \multicolumn{2}{|c|}{$\begin{array}{c}\text { c-axis distribution } \\
\Delta_{\theta_{50}} \text { (deg.) }\end{array}$} & 10 & 10 \\
\hline \multirow{3}{*}{$\begin{array}{c}\text { Exchange } \\
\text { coupling constant } \\
\left(\times 10^{-7} \mathrm{erg} / \mathrm{cm}\right)\end{array}$} & $A_{\text {intergrain }}$ & 1.2 & 0.5 \\
\hline & $A_{\text {intragrain }}$ & \multicolumn{2}{|c|}{10.0} \\
\hline & $A_{\text {interlayer }}$ & \multicolumn{2}{|c|}{$0.3-3.0$} \\
\hline
\end{tabular}

Table 2. Parameters of $H_{\mathrm{k}}$ for various layer thickness ratio.

\begin{tabular}{|c|c|c|c|}
\hline Layer thickness ratio & $1: 3$ & $1: 1$ & $3: 1$ \\
\hline$L_{\mathrm{s}}(\mathrm{nm})$ & 4 & 8 & 12 \\
\hline$L_{\mathrm{h}}(\mathrm{nm})$ & 12 & 8 & 4 \\
\hline$H_{\mathrm{k}}$ of Soft layer $(\mathrm{kOe})$ & 8 & 11 & 13 \\
\hline$H_{\mathrm{k}}$ of Hard layer $(\mathrm{kOe})$ & 22 & 25 & 30 \\
\hline
\end{tabular}

recording layer was divided into $5 \times 5 \times 2 \mathrm{~nm}^{3}$ cubic cells. Exchange lengths of the soft and hard layers are comparable to the cell size in this simulation. Table 1 shows parameters of the stacked media. The total thickness of the stacked media is $16 \mathrm{~nm}$ with varying the soft layer thickness $L_{\mathrm{s}}$ and the hard layer thickness $L_{\mathrm{h}}$. The layer thickness ratio of the soft and hard layers was 1:3 $\left(L_{\mathrm{s}}=4 \mathrm{~nm}\right), 1: 1\left(L_{\mathrm{s}}=8 \mathrm{~nm}\right)$ and $3: 1\left(L_{\mathrm{s}}=12 \mathrm{~nm}\right)$. The interlayer exchange coupling constant $A_{\text {interlayer }}$ was varied from $0.3 \times 10^{-7}$ to $3.0 \times 10^{-7} \mathrm{erg} / \mathrm{cm}$. The coercivity of these stacked media with each thickness ratio was adjusted to about $10 \mathrm{kOe}$. Table 2 shows parameters of anisotropy field $H_{\mathrm{k}}$ for various layer thickness ratio. The hysteresis loop of each stacked medium is set to almost the same figure by varying $H_{\mathrm{k}}$. The time-evolutional magnetization reversal process in the soft and hard layers was analyzed during application of printing field $H_{\mathrm{a}}$ and after removal of the $H_{\mathrm{a}}$. Printing performance PP was evaluated from the calculated magnetization distribution in the soft and hard layers. The PP in each layer was estimated by using the following definition [9]:

$$
\operatorname{PP}(\%)=\frac{\sum M_{z}^{\text {ideal }} M_{z}^{\text {cal }}}{\sum M_{z}^{\text {ideal }} M_{z}^{\text {ideal }}} \times 100,
$$

where $M_{\mathrm{z}}^{\text {ideal }}$ and $M_{\mathrm{z}}^{\text {cal }}$ are $z$-component of ideally printed magnetization and $z$-component of calculated magnetization, respectively. The PP means whether the calculated magnetization is close to the ideal magnetization. When the printed magnetization is ideal, the value of PP is $100 \%$.

\section{Results and discussion}

Fig. 3 shows magnetization distribution during applying the $H_{\mathrm{a}}$ for the $L_{\mathrm{s}}$ of $4 \mathrm{~nm}$ and the $A_{\text {interlayer }}$ of $1.8 \times 10^{-7}$ $\mathrm{erg} / \mathrm{cm}$. Figs. 3(a), (b) show the magnetization distribution of each layer for elapsed time of $70 \mathrm{ps}$ and
636 ps after applying the $H_{\mathrm{a}}$ of $4.5 \mathrm{kOe}$, respectively. Here the $H_{\mathrm{a}}$ of $4.5 \mathrm{kOe}$ is the optimum printing field in this case [10]. In Fig. 3, white area represents $M_{\mathrm{z}} / M_{\mathrm{s}}=1$, and black area represents $M_{\mathrm{z}} / M_{\mathrm{s}}=1$. When the $H_{\mathrm{a}}$ is applied, the magnetization is reversed first in the soft layer as shown in Fig. 3(a). Then, the magnetization reversal of the hard layer is induced by the magnetization of the soft layer as shown in Fig. 3(b). Fig. 4 shows the time-evolutional PP with a lapse of time in the soft and hard layers for the $L_{\mathrm{s}}$ of $4 \mathrm{~nm}$ and the $A_{\text {interlayer }}$ of $1.8 \times 10^{-7}$ $\mathrm{erg} / \mathrm{cm}$. It is found that during applying the $H_{\mathrm{a}}$ the magnetization of the soft layer is printed first in accordance with the pattern of master, and that magnetization reversal of the hard layer follows slightly behind that of the soft layer. This magnetization reversal process is equivalent to incoherent rotation [3].

Fig. 5 shows magnetization distribution during applying the $H_{\mathrm{a}}$ for the $L_{\mathrm{s}}$ of $8 \mathrm{~nm}$ and the $A_{\text {interlayer }}$ of $1.8 \times 10^{-7} \mathrm{erg} / \mathrm{cm}$. Figs. $5(\mathrm{a})$, (b) show the magnetization distribution of each layer for elapsed time of $70 \mathrm{ps}$ and $641 \mathrm{ps}$ after applying the $H_{\mathrm{a}}$ of $2.5 \mathrm{kOe}$, respectively. Fig. 6 shows the time-evolutional PP with a lapse of time in the soft and hard layers for the $L_{\mathrm{s}}$ of $8 \mathrm{~nm}$ and the $A_{\text {interlayer }}$ of $1.8 \times 10^{-7} \mathrm{erg} / \mathrm{cm}$. Although much of the magnetization

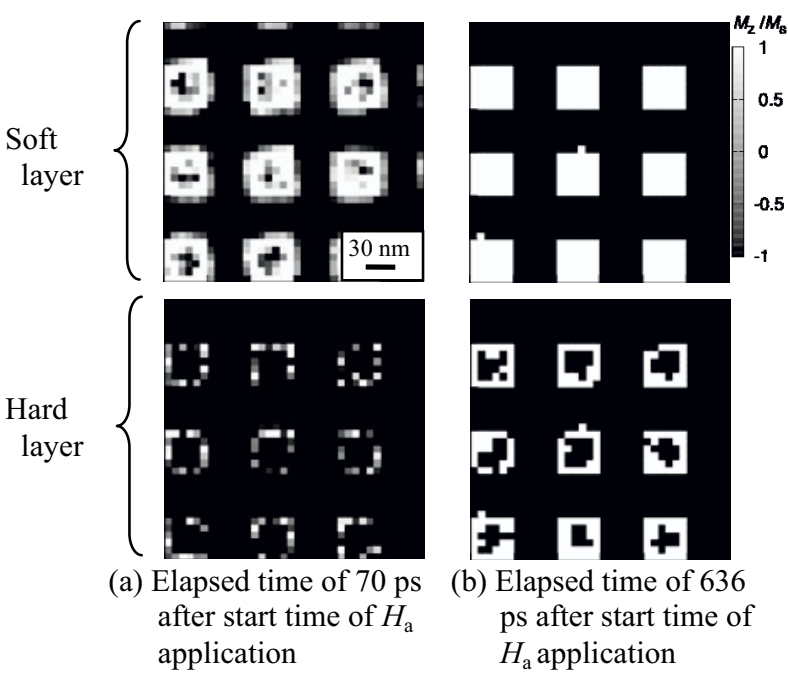

Fig. 3. Change in magnetization with a lapse of time. Top figures indicate magnetization distribution in soft layer, and bottom figures indicate that in hard layer $\left(L_{\mathrm{s}}=4 \mathrm{~nm}, A_{\text {interlayer }}=\right.$ $\left.1.8 \times 10^{-7} \mathrm{erg} / \mathrm{cm}\right)$.

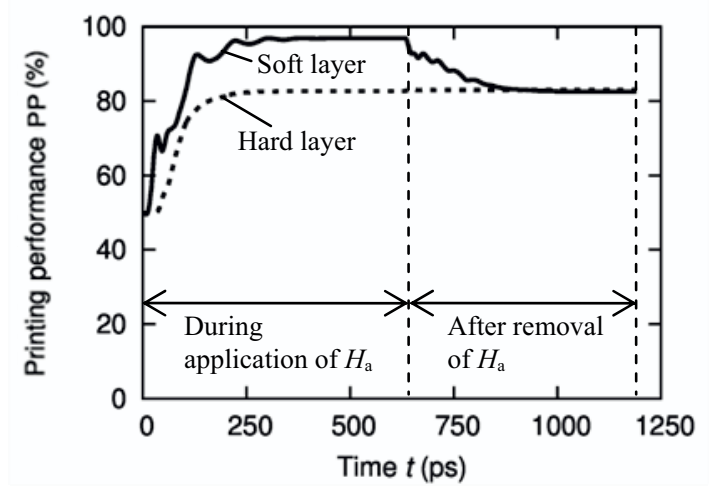

Fig. 4. Printing performance with a lapse of time in soft and hard layers $\left(L_{\mathrm{s}}=4 \mathrm{~nm}, A_{\text {iterlyaer }}=1.8 \times 10^{-7} \mathrm{erg} / \mathrm{cm}, H_{\mathrm{a}}=4.5 \mathrm{kOe}\right)$. 


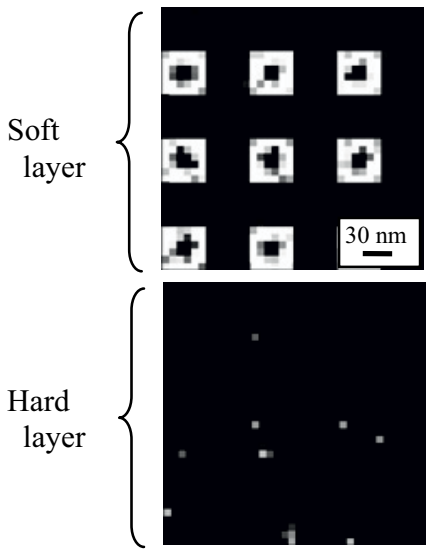

(a) Elapsed time of $70 \mathrm{ps}$ after start time of $H_{\mathrm{a}}$ application

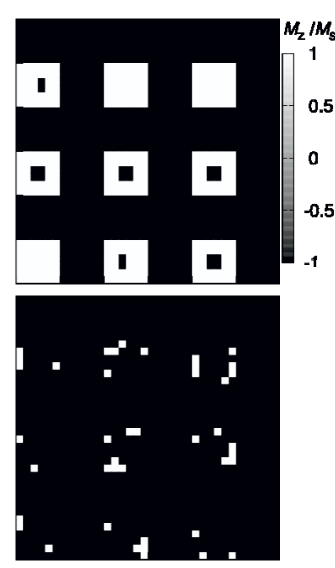

(b) Elapsed time of 641 ps after start time of $H_{\mathrm{a}}$ application

Fig. 5. Change in magnetization with a lapse of time. Top figures indicate magnetization distribution in soft layer, and bottom figures indicate that in hard layer $\left(L_{\mathrm{s}}=8 \mathrm{~nm}, A_{\text {interlayer }}=\right.$ $\left.1.8 \times 10^{-7} \mathrm{erg} / \mathrm{cm}\right)$.

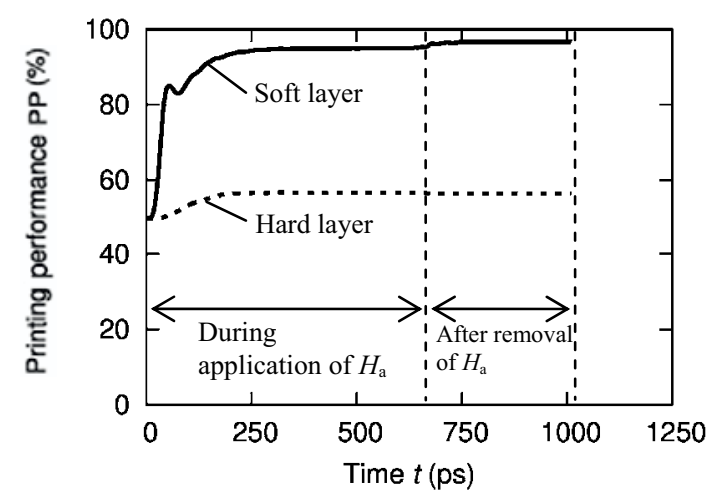

Fig. 6. Printing performance with a lapse of time in soft and hard layers $\left(L_{\mathrm{s}}=8 \mathrm{~nm}, A_{\text {iterlyaer }}=1.8 \times 10^{-7} \mathrm{erg} / \mathrm{cm}, H_{\mathrm{a}}=2.5 \mathrm{kOe}\right)$.

of the soft layer is reversed during applying the $H_{\mathrm{a}}$, magnetization in the hard layer hardly changes as shown in Figs. 5, 6. Namely the magnetization of each layer is independently reversed. This magnetization reversal process is equivalent to spin-flop rotation [3].

Fig. 7 shows magnetization distribution during applying the $H_{\mathrm{a}}$ for the $L_{\mathrm{s}}$ of $12 \mathrm{~nm}$ and the $A_{\text {interlayer }}$ of $1.8 \times 10^{-7} \mathrm{erg} / \mathrm{cm}$. Figs. 7(a), (b) show the magnetization distribution of each layer for elapsed time of $70 \mathrm{ps}$ and 743 ps after applying the $H_{\mathrm{a}}$ of $3.5 \mathrm{kOe}$, respectively. Fig. 8 shows the time-evolutional PP as a function of a lapse of time in the soft and hard layers for the $L_{\mathrm{s}}$ of $12 \mathrm{~nm}$ and the $A_{\text {interlayer }}$ of $1.8 \times 10^{-7} \mathrm{erg} / \mathrm{cm}$. When $H_{\mathrm{a}}$ is applied, the magnetization is reversed first in the soft layer, and then the magnetization reversal of the hard layer is induced by the magnetization of the soft layer in the same way as the medium with the $L_{\mathrm{s}}$ of $4 \mathrm{~nm}$. This magnetization reversal process corresponds to incoherent rotation.

Herein, the $\mathrm{PP}_{\max }^{\mathrm{s}}$ and $\mathrm{PP}_{\max }^{\mathrm{h}}$ are defined as the maximum values of the PP of the soft layer and the hard layer as shown in Fig. 8, respectively. Fig. 9 shows dependence of the $\mathrm{PP}_{\max }{ }^{\mathrm{s}}$ and the $\mathrm{PP}_{\max }{ }^{\mathrm{h}}$ on the $L_{\mathrm{s}}$ for the $A_{\text {interlayer }}$ of $1.8 \times 10^{-7} \mathrm{erg} / \mathrm{cm}$. Due to application of the optimum printing field, the $\mathrm{PP}_{\max }{ }^{\mathrm{s}}$ obtains high values for

all $L_{\mathrm{s}}$. On the other hand, the $\mathrm{PP}_{\max }{ }^{\mathrm{h}}$ obtains high values for the $L_{\mathrm{s}}$ of 4 and $12 \mathrm{~nm}$. When the $L_{\mathrm{s}}$ is $8 \mathrm{~nm}$, the $\mathrm{PP}_{\max }{ }^{\mathrm{h}}$ is minimum. This issue will be discussed as follow.

$H_{\text {total }}$ is magnetic field to reverse the magnetization of the hard layer, expressed by

$$
\boldsymbol{H}_{\text {total }}=\boldsymbol{H}_{\mathrm{ex}}+\boldsymbol{H}_{\mathrm{d}}+\boldsymbol{H}_{\mathrm{r}},
$$

where $H_{\mathrm{ex}}, H_{\mathrm{d}}$ and $H_{\mathrm{r}}$ are exchange field, magnetostatic

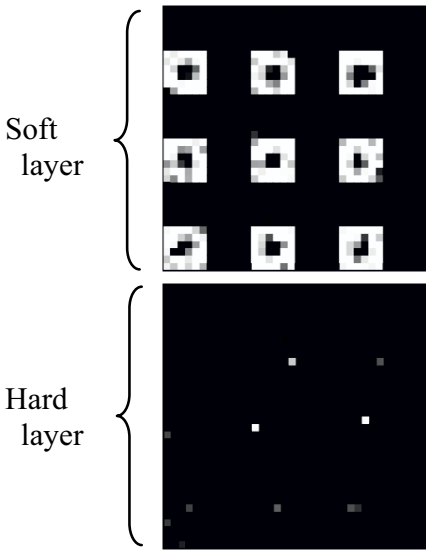

(a) Elapsed time of $70 \mathrm{ps}$ after start time of $H_{\mathrm{a}}$ application
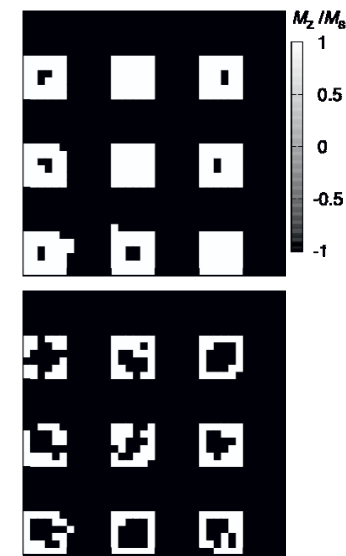

(b) Elapsed time of 743 ps after start time of $H_{\mathrm{a}}$ application
Fig. 7. Change in magnetization with a lapse of time. Top figures indicate magnetization distribution in soft layer, and bottom figures indicate that in hard layer $\left(L_{\mathrm{s}}=12 \mathrm{~nm}, A_{\text {interlayer }}=\right.$ $\left.1.8 \times 10^{-7} \mathrm{erg} / \mathrm{cm}\right)$.

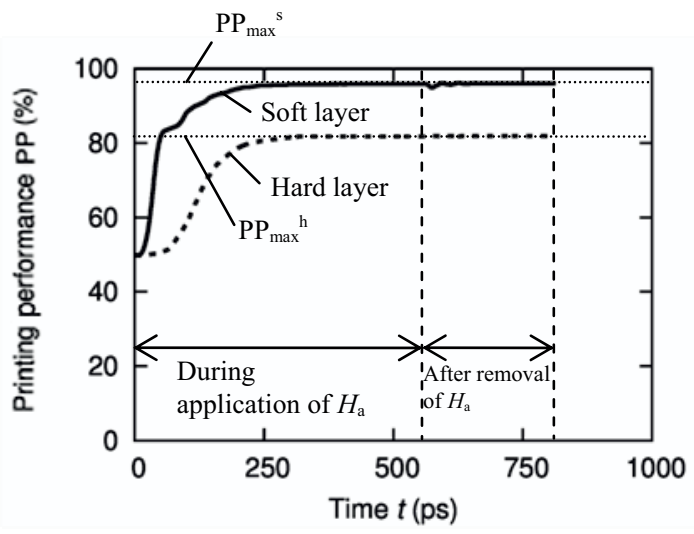

Fig. 8. Printing performance with a lapse of time in soft and hard layers $\left(L_{\mathrm{s}}=12 \mathrm{~nm}, A_{\text {iterlyaer }}=1.8 \times 10^{-7} \mathrm{erg} / \mathrm{cm}, H_{\mathrm{a}}=3.5\right.$ $\mathrm{kOe})$.

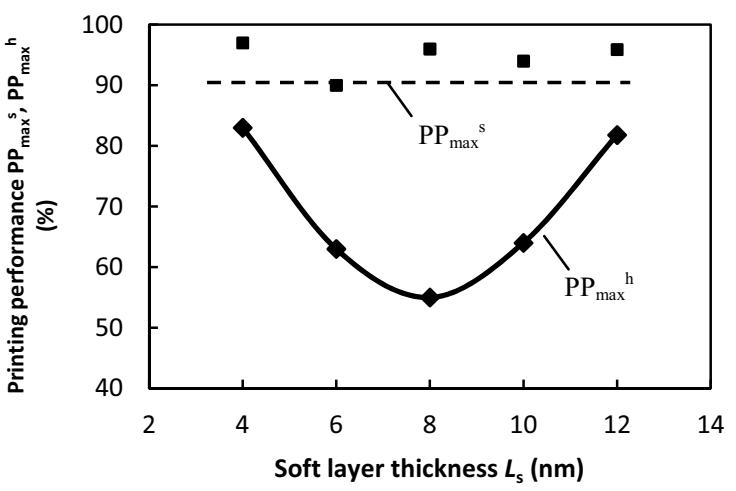

Fig. 9. Dependence of printing performance of each layer on soft layer thickness $L_{\mathrm{s}}\left(A_{\text {interlayer }}=1.8 \times 10^{-7} \mathrm{erg} / \mathrm{cm}\right)$. 
field and recording field, respectively. In magnetization reversal process, the anisotropy field $H_{\mathrm{k}}$ is considered to be applied to the opposite direction of the $H_{\text {total }}$. The magnetization reversal occurs when the $H_{\text {total }}$ becomes larger than the $H_{\mathrm{k}}$. For the medium with the $L_{\mathrm{s}}$ of $4 \mathrm{~nm}$, the $H_{\mathrm{r}}$ applied to the hard layer is strong due to small spacing between the master and the hard layer. Therefore, it is inferred that the $H_{\text {total }}$ becomes large and the hard layer has high $\mathrm{PP}_{\max }{ }^{\mathrm{h}}$. For the $L_{\mathrm{s}}$ of $12 \mathrm{~nm}$, although the $H_{\mathrm{r}}$ is not so strong and the $H_{\mathrm{ex}}$ is almost the same as that of the medium with the $L_{\mathrm{s}}$ of $4 \mathrm{~nm}$, the hard layer is easy to reverse due to a small grain volume. Therefore, it is inferred that the hard layer has high $\mathrm{PP}_{\max }{ }^{\mathrm{h}}$. On the other hand, for the $L_{\mathrm{s}}$ of $8 \mathrm{~nm}$, because the $H_{\mathrm{r}}$ is not so strong as that of the medium with the $L_{\mathrm{s}}$ of $4 \mathrm{~nm}$ and the volume of a grain of the hard layer is not so small as that of the medium with the $L_{\mathrm{s}}$ of $12 \mathrm{~nm}$, it is inferred that the $\mathrm{PP}_{\max } \mathrm{h}$ of the hard layer is low.

In order to discuss the magnetization reversal in the soft and hard layers, $\mathrm{PP}_{\max }{ }^{\mathrm{s}-\mathrm{h}}$ is defined as $\mathrm{PP}_{\max }{ }^{\mathrm{s}-\mathrm{h}}=$ $\mathrm{PP}_{\max }{ }^{\mathrm{s}}-\mathrm{PP}_{\max }{ }^{\mathrm{h}}$. For the $\mathrm{PP}_{\max }{ }^{\mathrm{s}-\mathrm{h}}$ higher than about $40 \%$, the magnetization of only the soft layer is reversed, namely magnetization reversal process is the spin-flop rotation. For the $\mathrm{PP}_{\max }{ }^{\mathrm{s}-\mathrm{h}}$ is less than about $5 \%$, the magnetization in the each layer is reversed almost simultaneously, which is the coherent rotation process. The incoherent rotation which is suitable for the recording media is in the region between the spin-flop and the coherent rotation regions. Fig. 10 shows

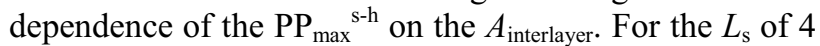
and $12 \mathrm{~nm}$, the magnetization reversal process is divided into the spin-flop region for $A_{\text {interlayer }}<$ about $1.3 \times 10^{-7}$ $\mathrm{erg} / \mathrm{cm}$, the incoherent region for about $1.3 \times 10^{-7}<$ $A_{\text {interlayer }}<$ about $2.2 \times 10^{-7} \mathrm{erg} / \mathrm{cm}$ and the coherent region for $A_{\text {interlayer }}>$ about $2.2 \times 10^{-7} \mathrm{erg} / \mathrm{cm}$. On the other hand, for the $L_{\mathrm{s}}$ of $8 \mathrm{~nm}$, the magnetization reversal process is divided into the spin-flop region for $A_{\text {interlayer }}<$ about $1.8 \times 10^{-7} \mathrm{erg} / \mathrm{cm}$, the incoherent region for about $1.8 \times 10^{-7}$ $<A_{\text {interlayer }}<$ about $2.5 \times 10^{-7} \mathrm{erg} / \mathrm{cm}$ and the coherent region for $A_{\text {interlayer }}>$ about $2.5 \times 10^{-7} \mathrm{erg} / \mathrm{cm}$. Above mentioned results show that the magnetization reversal process depends on the layer thickness ratio, and that the $A_{\text {interlayer }}$ to get the incoherent rotation has to be set to larger value for the media with the ratio near $1: 1$.

\section{Conclusion}

In this study, we investigated the effect of the layer thickness ratio of the soft layer to the hard layer and the interlayer exchange coupling on the time-evolutional magnetization reversal process in the stacked media with high coercivity by utilizing micromagnetic simulation. The results are as follows. Regardless of the layer thickness ratio, for each stacked medium the magnetization reversal process is divided into three regions, namely the spin-flop rotation, the incoherent rotation and the coherent rotation along with increase of the interlayer exchange coupling constant $A_{\text {interlayer }}$ In order to get the incoherent rotation region which is suitable for the recording media, it is required that the $A_{\text {interlayer }}$ is between about $1.3 \times 10^{-7}$ and $2.2 \times 10^{-7} \mathrm{erg} / \mathrm{cm}$

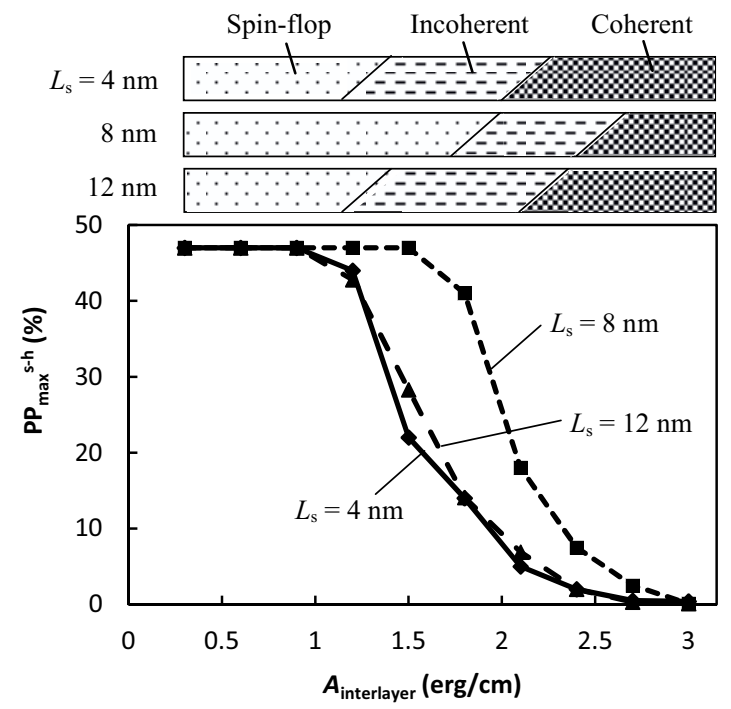

Fig. 10. Dependence of $\mathrm{PP}_{\max }{ }^{\mathrm{s}-\mathrm{h}}$ on $A_{\text {interlayer }}$ for various soft layer thickness $L_{\mathrm{s}}$.

for the media with the layer thickness ratio of $1: 3$ and 3 : 1 , and that one is between about $1.8 \times 10^{-7}$ and $2.5 \times 10^{-7}$ $\mathrm{erg} / \mathrm{cm}$ for the media with the ratio near $1: 1$.

\section{Acknowledgment}

This work was supported in part by the Grant-in-Aid for Scientific Research (C) (No. 24560394) from the Japan Society for the Promotion of Science (JSPS) of Japan.

\section{References}

1. R. H. Victora, and X. Shen: IEEE Trans. Magn., 41, 2828 (2005).

2. Y. Shiroishi, K. Fukuda, I. Tagawa, H. Iwasaki, S. Takenoiri, H. Tanaka, H. Mutoh, and N. Yoshikawa: IEEE Trans. Magn., 45, 3816 (2009).

3. Y. Inaba, T. Shimatsu, S. Watanabe, O. Kitakami, S. Okamoto, H. Muraoka, H. Aoi, and Y. Nakamura: J. Magn. Soc. Jpn., 31, 178 (2007).

4. A. Berger, N. Supper, Y. Ikeda, B. Lengsfield, A. Moser, and E. E. Fullerton: Appl. Phys. Lett., 93, 122502 (2008).

5. A. Oyama, T. Komine, and R. Sugita: Eur. Phys. J. Woc., 40, 07003 (2013).

6. A. Oyama, T. Komine, and R. Sugita: J. Magn. Soc. Jpn., 37, 62 (2013).

7. S. Greaves, Y. Kanai, and H. Muraoka: IEEE Trans. Magn., 45, 3823 (2009).

8. N. Sheeda, M. Nakazawa, H. Konishi, T. Komine, and R. Sugita: IEEE Trans. Magn., 45, 3676 (2009).

9. T. Komine, T. Murata, Y. Sakaguchi, and R. Sugita: IEEE Trans. Magn., 44, 3416 (2008).

10. A. Izumi, Y. Nagahama, T. Komine, R. Sugita, and T. Muranoi: J. Magn. Soc. Jpn., 30, 184 (2006). 